\title{
Homöopathieforschung: Problematik und Ergebnisse zur Wirksamkeit - mit Resultaten aus dem Programm Evaluation Komplementärmedizin PEK
}

\author{
Marco Righetti \\ Praxis für Homöopathie, $\mathrm{CH}$-Zürich
}

D as Programm Evaluation Komplementärmedizin (PEK, 1999-2005) des Schweizer Bundesamtes für Gesundheitswesen (BAG, zuvor BSV) hatte zum Ziel, Grundlagen über die Wirksamkeit, Zweckmässigkeit und Wirtschaftlichkeit (WZW) der ärztlichen Komplementärmedizin zu liefern im Hinblick auf den Entscheid über deren definitiven Verbleib in der Grundversicherung. Das PEK-Projekt war schon $\mathrm{zu}$ Beginn [1], aber vor allem in der Schlussphase, gekennzeichnet von enormen Mängeln und Unkorrektheiten, die sich zum Beispiel in Stellungnahmen eines Mitglieds des Lenkungsausschusses [2, 3] sowie des Review Boards [4] artikulierten: Der Datenzugang wurde behindert, die Resultate durften nicht präsentiert werden, der Schlussbericht wurde zensuriert, Ergebnisse vor allem über die Praxisstudien sind bis heute unvollständig, fehlen oder wurden nicht publiziert [5, 6]. Das Thema soll hier nicht weiter diskutiert werden. Zukünftigen politischen Entscheidungen ist zu wünschen, dass sie „entsprechend der vorhandenen wissenschaftlichen Information und nicht entsprechend vorgefasster Meinungen“ gefällt werden [7].

Wie waren die Schweizer Homöopathieärzte am PEK beteiligt? Bei der „Lancet-Studie“ des Instituts für Sozial- und Präventivmedizin ISPM Bern unter Professor EGGER [8] wurde den Homöopathen die Mitarbeit und lange sogar die Einsicht verwehrt [9, 10]. Beim HTA-Bericht (Health Technology Assessment) über die Homöopathie $[11,12,13]$ waren sie in Teilbereichen als Co-Autoren mitbeteiligt. Viele Homöopathieärzte haben zudem an der PEK-Praxisstudie teilgenommen.
Hintergrund: Das Programm Evaluation Komplementärmedizin PEK der Schweizer Regierung hatte zum Ziel, Daten über die Wirksamkeit, Zweckmässigkeit und Wirtschaftlichkeit der Komplementärmedizin zu liefern. In der Schlussphase litt das Projekt an verschiedenen Unzulänglichkeiten. Im Bereich Homöopathie wurden die Forschungsliteratur und die Praxis der Schweizer Ärzte untersucht. Probleme: Das grösste Hindernis für die Akzeptanz der Homöopathie sind die Hochpotenzen jenseits der Molekulargrenze und die unbekannte Wirkungsweise. Vom Anfang an basiert die Homöopathie auf empirischer Forschung, doch für den Entscheid über ihre Wirkung fordert man heute konventionelle Forschung. Homöopathie ist eine individuelle Methode und kann nicht in standardisierte Forschungsmuster gepresst werden. Fast alle Homöopathiestudien missachten diese Grundregeln und ermangeln praktischer Relevanz und externer Validität. Ergebnisse und Schlussfolgerungen: Unter den experimentellen Studien weisen trotz dieser Probleme der Basophilendegranulationstest und Tierintoxikationsstudien die beste Evidenz auf. Der Health Technology Assessment (HTA) des PEK untersuchte klinische Studien: 20 von 22 Systematischen Reviews zeigten positive Ergebnisse für die Homöopathie, darunter alle grossen rigorosen Metaanalysen. Dieses Resultat wird gestützt durch weitere grosse Übersichten, die im HTA nicht untersucht wurden. In der Domäne "Upper Respiratory Tract Infections/Allergies" (URTI/A) waren 24 von 29 Studien positiv. In ihrer "Lancet-Studie“ fanden Egger und das Institut für Sozial- und Präventivmedizin ISPM Bern gleichermassen positive Resultate in 110 homöopathischen und 110 gepaarten konventionellen Studien (Funnel plot-Analyse). Erst nach einer zweifelhaften Reduktion und Extrapolation aus 8 homöopathischen und 6 konventionellen Studien wurde das Ergebnis für die Homöopathie negativ. Die "Lancet-Studie" ist weder repräsentativ noch transparent und die Studienauswahl ist inadäquat. Weitere Mängel werden hier diskutiert. Ein angemessenerer Zugang zur Wirksamkeitsforschung in der Homöopathie sind grosse Outcome-Studien mit Langzeitbeobachtung unter realen Praxisbedingungen. Sie zeigen eine substanzielle praktische Effektivität und Nützlichkeit der Homöopathie. Der Artikel liefert einen Ausblick und Vorschläge zur zukünftigen Forschung und Evaluation der Homöopathie.

Schlüsselwörter: Homöopathieforschung, Problematik, klinische Ergebnisse, PEK-Studien, zukünftige Forschung

\section{Homoeopathic Research: Problems and Results on Effectiveness including Data from the „Programm Evaluation Komplementärmedizin PEK"}

Background: The "Programm Evaluation Komplementärmedizin PEK" (Program for the evaluation of complementary medicine) of the Swiss government was set up to provide data on effectiveness, appropriateness and cost-effectiveness of complementary medicine. Towards its end, the project suffered from various shortcomings. In the field of homoeopathy, research literature and the practice of Swiss doctors were examined. Problems: The biggest obstacles for the acceptance of homoeopathy are high potencies beyond the molecular limit and the unknown mode of action. From its beginning, homoeopathy was based on empirical research yet conventional research is currently demanded to decide whether it works. Homoeopathy is an individual method and cannot be forced into standardised research patterns. Nearly all homeopathic trials disregard these basic rules and are lacking in practical relevance and external validity. 
Results and Conclusion: Despite these problems, among experimental studies the basophil degranulation tests and animal intoxication studies demonstrate the best evidence. The Health Technology Assessment (HTA) of the PEK examined clinical studies: 20 of 22 Systematic Reviews showed positive results for homoeopathy, among them all the large rigorous meta-analyses. This result is supported by other large surveys not evaluated in the HTA. In the domain of "Upper Respiratory Tract Infections/ Allergies" 24 of 29 studies were positive. In their "Lancet-study" Egger and the Institute of Social and Preventive Medicine ISPM Berne found positive results in both groups, 110 homoeopathic and 110 matched conventional studies (funnel plot analysis). Only after a doubtful restriction and extrapolation from 8 homoeopathic and 6 conventional studies, did the result become negative for homoeopathy. The "Lancetstudy" is neither representative nor transparent and the study selection is inadequate. Further shortcomings are discussed here. A more appropriate approach to the assessment of homoeopathic effectiveness are large outcome studies with long-term observation under real practice conditions. They show a substantial practical effectiveness and utility of homoeopathy. The article provides an outlook und proposals for future research and evaluation of homoeopathy.

Key words: Homoeopathic research, problems, clinical results, PEK studies, future research

\section{Problematik der Homöopathieforschung}

Die grössten Probleme für die Anerkennung der Homöopathie sind die Hochpotenzen jenseits der Molekülgrenze und die unbekannte Wirkungsweise: Ist das ein Hokuspokus oder können die Arzneien wirken? Nach Kriterien der konventionellen Pharmakologie ist eine Wirkung unmöglich. Aber die Homöopathie hatte rasche Erfolge bei Epidemien, in der Behandlung von Millionen von Kranken, von schweren akuten und chronischen Krankheiten, von Tieren und Säuglingen. Ein Hokuspokus? Die Forschung soll's richten.

Homöopathie basiert von Anfang an auf empirischer Forschung. Aber diese zentrale systemimmanente Forschung zur Entwicklung der Methode interessiert angesichts des bedrohten Paradigmas niemanden ausserhalb der Homöopathie. Deshalb der Ruf nach konventioneller klinischer Forschung. Aber: Die Homöopathie beruht auf völlig individueller Mittelwahl, es gibt kein spezifisches Mittel für eine bestimmte Diagnose. Fast alle Homöopathiestudien missachten die Grundlagen und Grundregeln der Homöopathie, sind praxisfremd und meist ohne jegliche Praxisbedeutung. Das heisst, die externe Validität ist gering und die Gefahr falschnegativer Resultate gross. Mit andern Worten: Man zwängt die Homöopathie in ein unpassendes For- schungskleid und opfert die externe der internen Validität (externe Validität $=$ Gültigkeit für die reale Behandlungspraxis; interne Validität = innere, methodisch-statistische Gültigkeit der Studie). Besonders heikel und meist ungeeignet ist der "Goldstandard" der klinischen Forschung, die randomisierte kontrollierte Studie (RCT), welche die homöopathische Vorgehensweise verfälscht und sich auch bei anderen komplexeren Fragestellungen wenig bewährt hat.

\section{Resultate und Diskussion}

Seit Ende der 1980er-Jahre fehlt ein umfassender Literatur-Überblick über die Homöopathieforschung [14, 15). Im Rahmen des PEK wurde von den Homöopathieärzten schon zu Beginn eine umfassende Aufarbeitung der Literatur gefordert [1]: Diese wurde ihnen auch zugesagt, doch leider nur in sehr redimensionierter Form im Rahmen des HTA erfüllt.

Zum Wirkprinzip gibt es weitgehend nur Modelle und Hypothesen. In der Grundlagenforschung gibt es zwar Hinweise - aus NMR-/Kernspinresonanz- und UV-Spektrographie - auf dynamische Veränderungen des Lösungsmittels durch homöopathische Potenzen, doch ist diese Evidenz bisher nicht genügend robust und reproduzierbar [16, 17].

In zahlreichen Studien ist es trotz der genannten Probleme exemplarisch gelungen, die experimentelle Wirkung und klinische Wirksamkeit der Homöopathie nachzuweisen. Bei den experimentellen Studien wurde die Wirkung mit dem Basophilendegranulationstest (BDT) aus der Allergologie [18] und mit Tierintoxikationsstudien (Schutz und Entgiftung des Versuchstiers durch Potenzen des jeweiligen Gifts; Metaanalyse über 105 Studien [19]) am häufigsten und besten belegt und reproduziert. Viele dieser Studien stammen aus Pharmakologischen Fakultäten Frankreichs und bleiben seit Jahrzehnten weitgehend unbeachtet von den Juroren der Homöopathie. Eine grosse Mehrzahl der Studien mit diesen beiden Versuchsmodellen war positiv. Die BDT-Versuche von BENVENISTE [20] - in Nature publiziert - führten aber $\mathrm{zu}$ beträchtlichen Kontroversen. Auch andere experimentelle Ansätze zeigten positive Resultate, z.B. die Beeinflussung der Metamorphose von Amphibien mit potenziertem Thyroxin [21 u.a.]. Die Summe positiver Evidenz bei den experimentellen Studien ist beachtlich.

Bei der klinischen Beurteilung der Homöopathie sollte man die "weichen" Evidenzfakten nicht ganz ausser Acht lassen: die historische Evidenz, die Fallberichte und die 200 Jahre Praxis an Millionen von Kranken. Unter den klinischen Studien ist die Evidenzlage nach Mathie [22] bei 9 Diagnosen besonders gut, bei weiteren Diagnosen weniger eindeutig. Im HTA-Bericht Homöopathie des PEK [11, 12, 13] wurde nur der Bereich „Upper Respiratory Tract Infections/Allergies“ (URTI/A) untersucht: 24 von 29 evaluierten Studien zeigen ein positives Ergebnis (Signifikanz oder Trend) zu Gunsten der Homöopathie und belegen deren Wirksamkeit. 23 der 29 Studien sind kontrolliert. Hier lässt sich auch deutlich aufzeigen, wie schwierig und aufwändig die Literatursuche in der Homöopathie ist. Trotz erweiterter Suchstrategien über die indexierten Publikationen hinaus fand man nachträglich mehr als 20 weitere URTI/A-Studien [v.a. in 23]. Sie stützen mit ihrer deutlich positiven Evidenz das Ergebnis.

Bei den Übersichtsstudien zeigen im HTA 20 von 22 Systematischen Reviews (SR) ein positives Ergebnis, darunter 
alle grossen rigorosen schulmedizinischen Reviews von KLEIJNEN et al. [24], BoISSEL [25], Linde et al. [26] und Cucherat [27]. Weitere umfassende Metaanalysen und Übersichten mit einer grossen Fülle an positiver Evidenz wurden zwar als Datenquellen benützt, aber - weil nicht Systematische Reviews - im HTA gar nicht ausgewertet: Auch sie belegen die klinische Wirksamkeit der Homöopathie [z.B. 22, 23, 28 u.a.].

In ihrer „Lancet-Studie“ kommen Prof. EGGER und das Institut für Sozialund Präventivmedizin ISPM Bern zu anderen Ergebnissen [8]. Hier ist nicht der Ort, das unhaltbare Vorgehen der Autoren zu diskutieren, welche in den Medien fast eineinhalb Jahre lang mit der Schlagzeile „Homöopathie gleich Placebo“ die Geheimhaltungspflicht und interne PEK-Vereinbarungen verletzt haben, bevor die am PEK beteiligten Homöopathen die Studie im Januar 2005 endlich einsehen und später Stellung beziehen durften [9]. Die Studie vergleicht 110 homöopathische mit 110 gepaarten konventionellen (schulmedizinischen) Studien. Und was ist an der Studie dran? Sie ist nicht repräsentativ und nicht valid. Die Autoren können deshalb gar keine Aussage über Homöopathie machen:

1. Sie messen nicht die real praktizierte Homöopathie, sondern verwechseln diese mit verzerrter Studienhomöopathie. In der Praxis würde kaum je ein Patient so wie in einer dieser 110 Studien behandelt!

2. Die grosse Mehrzahl der Studien zeigt dennoch ein ähnlich positives Ergebnis wie die konventionelle Medizin.

3. Das negative Ergebnis beruht einzig auf einer Reduktion auf 8(!) grössere, willkürlich ausgewählte, meist praxisfremde Homöopathiestudien im Vergleich zu 6(!) konventionellen Studien und aus einer fragwürdigen statistischen Extrapolation. Auf welchen 8 Studien das negative Ergebnis beruhen soll, darüber schweigt die Lancet-Publikation! Erst Monate später sind diese auf der Website des Instituts einsehbar, ihre Auswahl wird aber nicht begründet.

4. Viele weitere Details der ISPM-Studie sind mangelhaft und nicht halt- bar: Die Analyse und die grafische Statistik sind völlig intransparent, Angaben und Bewertungen zu den einzelnen Studien fehlen völlig. Die Arbeit ist tendenziös: Es fehlen Hinweise auf die geringe externe Validität der untersuchten RCT-Studien und die Gefahr falschnegativer Resultate ebenso wie Hinweise auf die diametral entgegengesetzen Ergebnisse in anderen grossen Übersichten. Das Kriterium der Studiengrösse wird übergewichtet. Die Metaanalyse und grafische Statistik (Funnel Plot) ist ungeeignet für heterogene Studien wie im Fall der Homöopathie: Man kann nicht alles in einen Sammeltopf werfen. Die Studienauswahl ist unvollständig und mangelhaft: 60 Studien werden ohne nähere Begründung ausgeschlossen. Allein bei KLEIJNEN et al. [24] und WEIN [23] finden sich zusammen rund 250 RCT-Studien, etwa drei Viertel davon mit positivem Ergebnis. Die Autoren haben demnach die kontrollierten Homöopathiestudien bei weitem nicht so vollständig erfasst, wie sie glauben. Sie widersprechen damit ihren eigenen Kriterien [29] und jenen der „Cochrane Collaboration“. Mit ihrer Reduktion auf 8 Studien entwerten sie ihre eigene Funnel plot-Methode und produzieren wohl selber einen „selection bias“. Trotz einer Mehrzahl positiver RCT-Studien entsteht so das negative Ergebnis.

Die Aussage „Homöopathie gleich Placebo“ ist wissenschaftlich unhaltbar. Der Leserbrief des SVHA wurde wie viele andere von Lancet zurückgewiesen, aber als „Offener Brief“ vielerorts publiziert [30]. Zahlreiche kritische Stellungnahmen sind anderweitig erschienen [z.B. in 31, 32]. Lancet hat im Editorial „das Ende der Homöopathie“ deklariert - oder ist es das Ende der biomedizinischen Reliabilität?

Wie sind die Ergebnisse der Homöopathiestudien zu bewerten? Die Frage erinnert an das Problem des halbvollen oder halbleeren Glases. Aus streng homöopathischer Sicht könnte man die Existenz der Studien weitgehend ignorieren - aber die Studien existieren nun mal. Fast alle Studien sind wegen fehlender Modell- und externer Validität praxisfremd und im Gegensatz zu schulmedizinischen Studien für die praktische Arbeit meist bedeutungslos und uninteressant. Dazu kommt, dass in der Homöopathie Gelder, Forschungsinfrastruktur und eine potente Pharmaindustrie weitgehend fehlen. Angesichts dieser Fakten ist die grosse Mehrzahl positiver Wirkungs- und Wirksamkeitsnachweise doch erstaunlich und beachtlich. Aus dieser Sicht kann man sagen: Das Glas ist mindestens halbvoll. Umgekehrt wurden in Homöopathiestudien immer wieder unterschiedliche Modelle verwendet, relativ wenige Studien reproduziert und die gesamte Studienzahl ist - verglichen mit der Schulmedizin - klein, was angesichts der geringen praktischen Bedeutung auch nicht erstaunt. In diesem Sinn ist das Glas halbleer. Allerdings müsste auch bei kritischer Sichtweise gelten: „Absence of evidence is not evidence of absence“ [33] - zumal bei inadäquaten Forschungsansätzen.

Was wäre besser? Die grosse Mehrzahl der heute vorliegenden Studien zwängt die Homöopathie in ein inadäquates Forschungskorsett. Das homöopathische Wirkprinzip ist unbekannt: Aufgrund der Beobachtung in der Praxis besitzen Homöopathika im Gegensatz zu Allopathika keine spezifische Wirksamkeit gegen bestimmte Krankheiten, sondern vermutlich unspezifische Wirkungen auf Regulation und Selbstheilung und individuell-spezifische Wirkungen im jeweiligen Individuum. Deshalb ist wohl eher die Wirkung im realen Kontext als die pharmakologische Wirkung per se messbar. Die Untersuchungsmethode der Wahl, welche Individualisationsprinzip und Methodik der Homöopathie angemessen berücksichtigt, ist die Einzelfallstudie - zu Studienzwecken an genügend grossen Patientenzahlen und über genügend lange Zeit. In den letzten Jahren wurden denn auch vermehrt praxisnähere Studien durchgeführt, welche die klinische Wirksamkeit der Homöopathie in ihrer natürlichen Praxisumgebung bei akuten und chronischen Krankheiten aufzeigen können. Besonders wertvoll sind dabei grosse epidemiologische OutcomesStudien mit Langzeitbeobachtung [z.B. 
$34,35,36,37,38$ ], welche unter realen Praxisbedingungen eine ausgezeichnete und substanzielle klinische Wirksamkeit der Homöopathie nachweisen, darunter eine Studie an fast 4000 Patienten mit chronischen Krankheiten [36]. Auch die Ergebnisse der PEK-Praxisstudien gehen in diese Richtung [5, 6]. Ein Teil der Studien umfasst auch Kontrollgruppen mit konventioneller Behandlung. Die Homöopathie zeigt also das paradoxe Phänomen guter klinischer Effektivität und praktischen Nutzens bei weniger eindeutigen Resultaten in konventionellen Wirksamkeitsstudien (Wirksamkeitsparadox [39]).

\section{Zukünftige Forschung in der Homöopathie}

Einige Thesen (siehe auch [15]):

A. Ausgangslage und Adressat: Das historische Material mit raschen Erfolgen bei Epidemien [28] und die praktische Bewährung über 200 Jahre ist nur beschränkt aufgearbeitet, aber die „weichen Fakten“ sprechen für die Homöopathie. In der Praxis zeigt die Homöopathie, im Gegensatz zu Placebo, bei akuten bakteriellen Infekten sowie bei chronischen und rezidivierenden Krankheiten stabile (Langzeit-)Wirkung. Diese ist intraindividuell reproduzierbar bei richtiger, fehlt aber bei falscher Mittelwahl. Homöopathie ist nebenwirkungsarm und preiswert, zeigt in Outcomes-Studien und in der Mehrzahl experimenteller und klinischer Studien trotz geringer externer Validität positive Evidenz. Es stellt sich die Frage, was zum WZWNachweis überhaupt noch fehlt. Gesundheitsbehörden, Ökonomen, Patienten, Homöopathen, Hausärzte, klinische Pharmakologen oder medizinische Fakultäten würden diese Frage wohl unterschiedlich beantworten. Deshalb sieht auch das Forschungsdesign je nach Adressat, Zweck und Zielsetzung völlig verschieden aus. Auf alle Fälle ist es unerlässlich und von grösster Bedeutung, in jeder Studienveröffentlichung klar auf Problematik und Stellenwert der Forschungsmetho- dik und -ergebnisse hinzuweisen.

B. Geeignete stabile Forschungsinfrastrukturen an Universitäten oder qualifizierten Instituten und die aufwändige weltweite Sammlung und Aufarbeitung der bisherigen Forschungsergebnisse (auch aus der nicht indexierten Literatur) sind Grundvoraussetzungen für die zukünftige Forschung.

C. Zukünftige Forschung muss klar unterscheiden zwischen Forschung mit direktem Nutzen für den Fortschritt der Homöopathie und Forschung zum Zweck ihrer wissenschaftlichen und gesundheitspolitischen Anerkennung (,Rechtfertigungsstudien“). Die Forschung muss dabei die Besonderheiten der Homöopathie und schulmedizinische Kriterien gleichermassen berücksichtigen.

D. Die Verbesserung der homöopathischen Behandlungsqualität beruht in erster Linie auf der systemimmanenten Forschung (Arzneimittelprüfungen, Symptomen- und Verlaufsbeobachtung, Erweiterung von klinischer Erfahrung und Knowhow usw.). Hierher gehört zudem die Erforschung der Wirkungsweise, der natur- und geisteswissenschaftlichen Grundlagen, der Arzneiherstellung und -qualität sowie einiger epidemiologischer Fragen (siehe G). Die grossen Fragen von Gesundheit und Krankheit und ihrer integrativen Behandlung betreffen beide, Homöopathie und Schulmedizin.

E. Für den klinischen Wirksamkeitsnachweis ist in der Homöopathie die Einzelfallstudie (intraindividueller Versuch) mit individueller Mittelwahl am angemessensten - an genügend Patienten und bei chronischen Krankheiten über genügend lange Zeit. Für Forschungszwecke sind hier in erster Linie breit angelegte Beobachtungsstudien (OutcomeStudien) sinnvoll für eine verlässliche Datenerhebung über Praxis, Wirkung, Zweckmässigkeit und Kosten homöopathischer Behandlung unter unverfälschten, realen Praxisbedingungen, $d$.h. wenig beeinflusst durch methodenfremde Forschungsdesigns. Sie sollten Validitätskriterien der EBM (Evidenz-basierten
Medizin) wie der Homöopathie adäquat berücksichtigen und, falls möglich, auch Vergleiche mit Kontrollgruppen umfassen. Eine Variante wäre der Gesamtvergleich der Hausarztbehandlung eines homöopathischen mit einem schulmedizinischen Kollektiv über genügend lange Zeit, wie das von homöopathischer Seite im PEK vorgeschlagen wurde.

F. Randomisierte kontrollierte Studien (RCT) mit individueller Verordnung sollten nur nach genauer Studienplanung und Pilotphase durchgeführt werden, weil sie aus homöopathischer Sicht vor allem bei chronischen Krankheiten extrem aufwändig, schwierig und auch ethisch fragwürdig sind. Aus unserer Sicht macht es wenig Sinn, zu den wohl weit über 1000 experimentellen und klinischen Studien ohne klare Fragestellung willkürlich 2 bis 3 weitere Indikationsstudien hinzuzufügen. Ausnahmen erfordern in jedem Fall eine sorgfältige Studienplanung: Sie sind am ehesten denkbar bei akuten Krankheiten (Komplexität geringer) oder Sonderfällen (z.B. hyperaktive Kinder [40]).

G. RCT-Studien ohne individuelle Mittelwahl widersprechen den Grundlagen der Homöopathie und haben meist geringe externe Validität. Sie machen nur Sinn, falls mit einem Versuchsmodell ein prinzipieller und exemplarischer Wirksamkeitsnachweis erbracht werden kann. Eine gute Planung ist auch hier zentral. Sinnvoll wäre besonders die Reproduktion erfolgversprechender Studien, was bei geeignetem Setting zum Beispiel in der Traumatologie und bei allergischen Krankheiten möglich scheint. Gemeinsamer Nenner solcher Studien sind eng umschriebene Situationen mit möglicher Einschränkung des Individualitätsprinzips (standardisierte Therapie deckt evtl. die Mehrzahl der Fälle ab). Ein paar Fragestellungen nach eingeschränkter Individualität könnten dabei auch von homöopathischem und epidemiologischen Interesse ein: zum Beispiel in der Traumatologie, bei Insektenstichen und Allergien, in der Immunologie (Nosoden), bei Vergiftungen (Tierin- 
toxikationsmodell), bei sogenannt einseitigen Krankheiten.

H. Bei den experimentellen Studien (In vitro-, Tier-, Pflanzenstudien) gilt zum Teil Ähnliches wie bei G. Im Vordergrund steht die Etablierung weniger stabiler und reproduzierbarer Testsysteme, die trotz geringer externer Validität einen prinzipiellen Wirkungsnachweis erbringen können.

I. Grundlagenforschung: Hier geht es um auch für die Homöopathie interessante, zum Teil schwierig erforschbare Fragen nach dem Wirkprinzip, den physikalisch-chemischen (und geisteswissenschaftlichen?) Grundlagen und den Arzneiproblemen.

\section{Literatur}

1 Righetti M, SVHA: Homöopathie: Grundlagen, Anwendungsgebiete und mögliche Ansätze zu Forschungsstudien. Beitrag zum Programm Evaluation Komplementärmedizin PEK (Sept. 1999). Originalarbeit: Sekretariat SVHA; leicht modifiziert: www.homeodoctor.ch/righetti.htm oder www.svha.ch > Forschung > Grundlagenforschung

2 Heusser P: Medizin und Macht am Beispiel des Programms Evaluaiton Komplementärmedizin PEK 2005. www.svha.ch > Forschung

3 Heusser P: PEK und BAG: Probleme beim Programm Evaluation Komplementärmedizin. Schweiz. Ärztezeitung 2006;87:20:899-903

4 PEK Review Board: Summary Consensus Statement of the PEK Review Board regarding the PEK process and the PEK products, 27th September 2005. (englische und deutsche Version)

www.bag.admin.ch/kv/forschung/d/2005consensus_statement_review_board_d.pdf

5 PEK Schlussbericht 24.4.2005.

www.bag.admin.ch/themen/krankenversicherung/00305.02362/index.html (Hier finden sich Hinweise zu weiteren PEK-Publikation in Vorb.; Daten und Ergebnisse der Praxisstudien liegen zum Teil nur in Draft-Form für internen Gebrauch vor)

6 SVHA: Antrag des SVHA an die Eidgenössische Kommission für allgemeine Leistungen (ELK) in Sachen der definitiven Anerkennung der Klassischen Homöopathie als Pflichtleistung 31.3.2005 (überarbeitete Zusammenfassung unter dem Titel: Medizin optimieren Homöopathie integrieren. SVHA 2006)

7 Heusser $\mathrm{P}$, Walach $\mathrm{H}$ : Effektiv oder nicht? Entscheiden Sie selbst! - Die PEK-HTA-Berichte. Forsch Komplementarmed. 2006;13 (Suppl 2):2-3

8 Shang A, Huwiler K, Nartey L et al.: Are the clinical effects of homoeopathy placebo effects? Comparative study of placebo-controlled trials of homoeopathy and allopathy. The Lancet 2005;366:726-732

9 SVHA: Stellungnahme des Schweizerischen Vereins homöopathischer Arztinnen und Ärzte SVHA zur Homöopathiestudie des Instituts fü Sozial- und Präventivmedizin ISPM Bern ("Eggerstudie") vom 21. April 2005 (English version: Statement of the Swiss Association of the Homoeopathic Physicians on the homoeopathic study of the ...). www.svha.ch $>$ Forschung

10 Righetti M: Homöopathieforschung: Ergebnis se zur Wirksamkeit. 21.4.2005. www.svha.ch $>$ Forschung. English version: Righetti $M$ Homoeopathy research. Explore: J. Science Healing 2, 1, 2006: 8 (doi:10.1016/j.explore.2005.12.002). Or: Homoeopathy research: results on effectivenenss. www.svha.ch $>$ Forschung.

11 Bornhöft G, Wolf U, von Ammon K, Righetti $M$, Maxion-Bergemann S, Baumgartner S, Thurneysen A, Matthiessen P: Effectiveness, safety and cost-effectiveness of homeopathy in general practice - summarized health technology assessment. Forsch Komplementärmed. 2006:13 (Suppl 2):19-29. (HTA Homöopathie Kurzfassung, Englisch)

12 Bornhöft G, Matthiessen P (Hrsg.): Homöopathie in der Krankenversorgung - Wirksamkeit Nutzen, Sicherheit und Wirtschaftlichkeit. VAS, Frankfurt/Main 2006 (HTA-Bericht Homöopathie, PEK, Langversion, Deutsch)

13 HTA: Health Technology Assessment Berich Homöopathie, PanMedion Stiftung, PEK, Bundesamt für Gesundheitswesen Schweiz 2005 (siehe Bornhöft et al 2006: Kurz- und Langversion; kurze Zusammenfassung: www.bag.admin.ch/themen/krankenversicherung/00305.02362/index.html)

14 Poitevin B: Le Devenir de I 'Homéopathie Eléments de théorie et de recherche. Doin, Paris 1987

15 Righetti M: Forschung in der Homöopathie. Grundlagen, Problematik und Ergebnisse, Ulrich Burgdorf, Götingen 1988.

16 Becker-Witt C, Weisshuhn TER, Lüdtke R, Willich SN: Quality assessment of physical research in Homeopathy. J Alternative Complement Med 2003;9(1):113-132

17 Walach $H$, Jonas W B, Ives J, Van Wijk R, Weingärtner $\mathrm{O}$ : Research on Homeopathy: State of the Art. J Alternative and Complementary Medicine 2005;(11)5:813-829

18 Belon P, Cumps J, Ennis M, Mannaioni PF Roberfroid M, Sainte-Laudy J, Wiegant FA: Histamine dilutions modulate basophil activation. Inflamm Res. 2004;53(5):181-188

19 Linde K, Jonas WB, Melchart D et al.: Critical review and meta-analysis of serial agitated dilutions in experimental toxicology. Human and Experimental Toxicology, 1994;13:481-492

20 Davenas E et al.: Human basophil degranulation triggered by very dilute antiserum agains IgE. Nature 1988;333:816-818.

21 Endler PC, Ludtke R, Heckmann C, Zausner C Lassnig H, Scherer-Pongratz W, Haidvogl M Frass M: Pretreatment with thyroxine (10-(8) parts by weight) enhances a 'curative' effect of homeopathically prepared thyroxine (10 (13)) on lowland frogs. Forsch Komplementarmed Klass Naturheilkd. 2003;10(3):137-42

22 Mathie RT: The research ecidence base for homeopathy: a fresh assessment of the literature. Homeopathy 2003;92(2):84-91

23 Wein C: Qualitätsaspekte klinischer Studien zur Homöopathie. Karl und Veronica Carstens Stiftung KVC, Essen 2002

24 Kleijnen J, Knipschild P, ter Riet G: Clinical trials of homoeopathy. BMJ 1991;302:316-323

25 Boissel J. Critical Literature Review on the effectiveness of Homeopathy: Overview over data from homeopathic medicine trials. In: Commission of the European communities editor. Homeopathic Medicine Research Group. Commission of the European communities; 1996, 196-210

26 Linde K, Clausius N, Ramirez G, Melchart D, Eitel F, Hedges LV, Jonas WB: Are the effects of homeopathy placebo effects? A meta- analysis of randomised, placebo controlled trials. Lancet 1997:350:834-843

27 Cucherat M, Haugh MC, Gooch M, Boissel JP Evidence of clinical efficacy of homeopathy. A meta-analysis of clinical trials. HMRAG Homeopathic Medicines Research Advisory Group. Eur Clin Pharmacol 2000;56:27-33

28 Dean ME: The Trials of Homeopathy - Origins, Structure and Development. Karl und Veronica Carstens Stiftung KVC, Essen 2004

29 Smith G D, Egger M: Meta-analysis: Unresolved issues and future developments British Medical Journal, 1998: 221-225

30 SVHA: Open letter to the Editor of The Lancet from the Swiss Association of Homoeopathic Physicians (SVHA). Erschienen u.a. in Forsch Komplementärmed Klass Haturheilkd 2005: 12:352-353; Homeopathy 2006;95(1):61-62. www.svha.ch >Forschung (Englisch und Deutsch)

31 Journal of Alternative and Complementary Medicine Vol. 11, 5, 2005: zahlreiche Stellungnahmen zur Lancetstudie von Egger. www.liebertonline.com/toc/acm/11/5

32 Valeri A: La meta-analisi di Egger et al. sull'omeopatia permette di trarre conclusioni rilevanti per il sistema sanitaria? Un'analisi metodologica critica. www.omeomed.net, 2005

33 Altman DG, Bland M: Absence of evidence is not evidence of absence. Br Med J 1995: 311: 485

34 Güthlin $\mathrm{C}$, Lange $\mathrm{O}$, Walach $\mathrm{H}$ : Measuring the effects of acupuncture and homeopathy in general practice: an uncontrolled prospective documentation approach. BMC Public Health 2004;4:6

35 Becker-Witt C, Lüdtke R, Weisshuhn TER, Willich SN: Diagnoses and treatment in homeopathic medical practice. Forsch Komplementärmed Klass Naturheilkd 2004;11:98-103

36 Witt C, Lüdtke R, Baur R, Willich S N: Homeopathic medical practice: Long-term results of a cohort study with 3981 patients. BMC Public Health 2005; 5: 115, 1-8. www.biomedcentral.com/147-2458/5/115

37 Witt C, Keil T, Selim D et al: Outcome and costs of homoeopathic and conventional treatment strategies: A comparative cohort study in patients with chronic disorders. Complementary therapies in medicine 2005;13:79-86

38 Riley $D$, Fischer $M$, Singh $B$, Haidvogl $M$ Heger M: Homeopathy and conventional medicine: an outcome study comparing effectiveness in a primary care setting. J Alternative Complementary Med 2001;7(2):149-159

39 Walach H: Das Wirksamkeitsparadox in der Komplementärmedizin. Forsch Komplementärmedizin Klass Naturheilkd 2001;8:193-195

40 Frei $\mathrm{H}$ et al: Homeopathic treatment of children with attention deficite hyperactive disorder: a randomised, double blind, placebo controlled trial. 27.7.2005 http:// dx.doi.org/10.1007/s00431-005-1735-7.

\section{Korrespondenzadresse:}

Dr. med. Marco Righetti

Leonhardshalde 2, CH-8001 Zürich

m.righetti@bluewin.ch 